\title{
Charge transfer between epitaxial graphene and silicon carbide
}

\author{
Sergey Kopylov, ${ }^{1, a)}$ Alexander Tzalenchuk, ${ }^{2}$ Sergey Kubatkin, ${ }^{3}$ and Vladimir I. Fal'ko ${ }^{1}$ \\ ${ }_{1}^{1}$ Department of Physics, Lancaster University, LA1 4YB Lancaster, United Kingdom \\ ${ }^{2}$ National Physical Laboratory, TW11 OLW Teddington, United Kingdom \\ ${ }^{3}$ Department of Microtechnology and Nanoscience, Chalmers University of Technology, \\ S-412 96 Goteborg, Sweden
}

(Received 25 July 2010; accepted 18 August 2010; published online 16 September 2010)

\begin{abstract}
We analyze doping of graphene grown on $\mathrm{SiC}$ in two models which differ by the source of charge transferred to graphene, namely, from SiC surface and from bulk donors. For each of the two models, we find the maximum electron density induced in monolayer and bilayer graphene, which is determined by the difference between the work function for electrons in pristine graphene and donor states on/in $\mathrm{SiC}$, and analyze the responsivity of graphene to the density variation by means of electrostatic gates. (C) 2010 American Institute of Physics. [doi:10.1063/1.3487782]
\end{abstract}

Graphene ${ }^{1}$ - monolayer or bilayer of carbon atoms with a honeycomb lattice-is a gapless semiconductor, which can be used as a current-carrying element in field-effect transistors. It has been found that the epitaxial graphene grown onto centimeter-size wafers of the Si-terminated face of $\mathrm{SiC}$ (Refs. 2-4) maintains structural integrity over large area and demonstrates a relatively high mobility of carriers. ${ }^{5-7}$ This makes graphene synthesized on $\mathrm{SiC}(\mathrm{SiC} / \mathrm{G})$ a promising platform to build integrated electronic circuits, assuming one can control the carrier density in it. For transistor applications, bilayer graphene in $\mathrm{SiC} / \mathrm{G}$ is a particularly interesting material, since interlayer asymmetry (e.g., induced by a transverse electric field) opens a minigap in its spectrum. ${ }^{8-10}$

In this paper we present a theory of charge transfer from $\mathrm{SiC}$ to the epitaxial monolayer graphene (MLG) and bilayer graphene (BLG) grown on its surface. It has been noticed that epitaxial graphene is always substantially n-doped, so that use of $\mathrm{SiC} / \mathrm{G}$ in transistors requires reduction in carrier density using gates. ${ }^{4}$ The initial doping of graphene comes from a combination of bulk donors in $\mathrm{SiC}$ with the volume density $\rho$ and surface donor states with the sheet density of surface states, $\gamma(\mathrm{DoS})$. The charge transfer to graphene in a top-gated field-effect transistor can be found from solving the following two coupled equations:

$$
\begin{aligned}
& \gamma\left[A-4 \pi e^{2} d\left(n+n_{g}\right)-\varepsilon_{F}(n)\right]+\rho l=n+n_{g}, \\
& \tilde{A}=\varepsilon_{F}(n)+U+4 \pi e^{2} d\left(n+n_{g}\right) .
\end{aligned}
$$

Equation (1) accounts for the charge balance, with $n_{g}$ $=C V_{g} / e(e>0)$ being the areal density of electrons transferred to the gate. Here, $\widetilde{A} / A$ is the difference between the work function of graphene and the work function of electrons in the bulk/surface donors in $\mathrm{SiC}$, and $\varepsilon_{F}$ is the Fermi energy in doped graphene. Equation (2) describes the equilibrium between electrons in graphene and bulk donors, with $l$ standing for the depletion layer width in $\mathrm{SiC}, U$ $=2 \pi e^{2} \rho l^{2} / \chi$ being the height of the Schottky barrier ( $\chi$ is dielectric constant of $\mathrm{SiC}$ ), and $d$-the distance between the $\mathrm{SiC}$ surface and the middle of graphene layer (Fig. 1).

In the following, we calculate the density $n$ of electrons (for both MLG and BLG) in two limits: graphene doping

${ }^{a)}$ Electronic mail: sergkop@gmail.com. dominated by the charge transfer from (a) surface donors, which corresponds to solving Eq. (1) with $\rho l \rightarrow 0$ and (b) bulk donors (e.g., nitrogen), which corresponds to solving Eqs. (1) and (2) with $\gamma=0$. Charge transfer in a more generic situation, with arbitrary $\rho$ and $\gamma$, can be assessed by taking the largest of the two estimates. Then, we determine the responsivity factor, $r=-d n / d n_{g}$, which characterizes the ability to control the carrier density using external gates: $r \rightarrow 1$ would be optimal for transistor operation of gated $\mathrm{SiC} / \mathrm{G}$ devices whereas $r \ll 1$ would indicate that transistor operation is impossible.

MLG has linear spectrum $\varepsilon_{ \pm}(p)= \pm v p$, in the two valleys, corresponding to the nonequivalent corners $K$ and $K^{\prime}$ of the hexagonal Brillouin zone, so that $\varepsilon_{F}(n)=\hbar v \sqrt{\pi n}$ (we take into account both valley and spin degeneracy of the electron states). In the limit (a) we find that the carrier density is

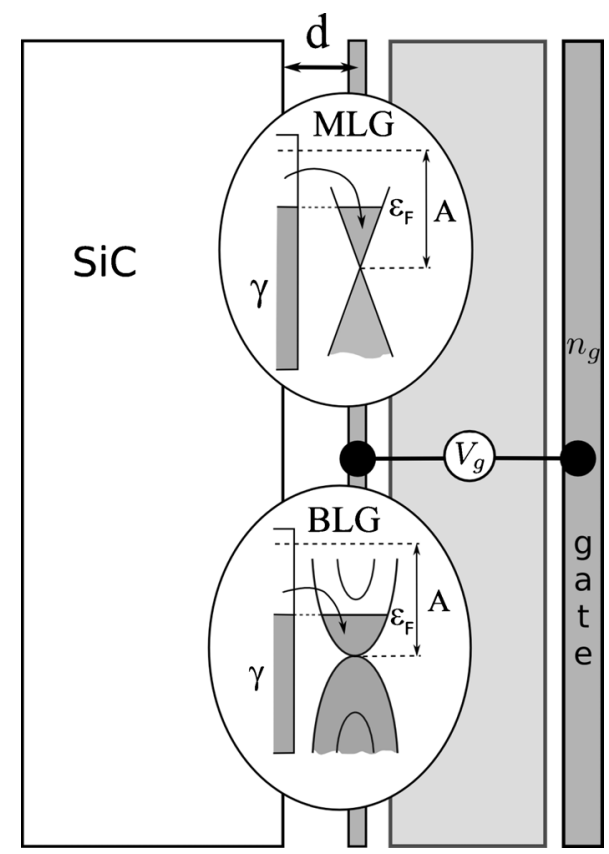

FIG. 1. Sketch of a SiC/G-based field effect transistor. Insets illustrate relevant part of the MLG and BLG band structure. 


$$
\begin{aligned}
& n^{I}=\frac{A \gamma^{2}\left[\sqrt{1+\frac{\gamma_{A}}{\gamma^{2}}\left(\gamma-\frac{n_{g}}{A} \frac{\gamma_{d}+\gamma}{\gamma_{d}}\right) \frac{\gamma_{d}+\gamma}{\gamma_{d}}}-1\right]^{2}}{\gamma_{A}\left(1+\frac{\gamma}{\gamma_{d}}\right)^{2}}, \\
& \gamma_{d}=\frac{1}{4 \pi d e^{2}}, \quad \gamma_{A}=\frac{4 A}{\pi \hbar^{2} v^{2}} .
\end{aligned}
$$

The initial density of electrons in graphene is described by Eq. (3) $\left(n_{g}=0\right)$ with two characteristic regimes,

$$
n^{I} \approx\left\{\begin{array}{cc}
A \gamma, & \gamma \ll \gamma_{*}^{I} \\
n_{*}^{I}, & \gamma \gg \gamma_{*}^{I}
\end{array},\right.
$$

where $n_{*}^{I}$ and $\gamma_{*}^{I}$ are, respectively, the saturation value for the carrier density in $\mathrm{SiC} / \mathrm{G}$ and the crossover value of DoS of donors on the $\mathrm{SiC}$ surface at which $n(\gamma)$ saturates, as follows:

$$
\gamma_{*}^{I}=\frac{\gamma_{A}}{\left(1+\sqrt{1+\frac{\gamma_{A}}{\gamma_{d}}}\right)^{2}}, \quad n_{*}^{I}=\frac{A \gamma_{A}}{\left(1+\sqrt{1+\frac{\gamma_{A}}{\gamma_{d}}}\right)^{2}} .
$$

The responsivity of MLG in $\mathrm{SiC} / \mathrm{G}$ is, then,

$$
\begin{aligned}
\left.r\right|_{n_{g}=0} & =1-\left(1+\frac{\gamma_{A}}{\gamma_{d}}+\frac{\gamma_{A}}{\gamma}\right)^{-1 / 2} \\
& \approx\left\{\begin{array}{cc}
1, & \gamma \ll \gamma_{*}^{I} \\
1-\frac{1}{\sqrt{1+\gamma_{A} / \gamma_{d}},} & \gamma \gg \gamma_{*}^{I}
\end{array} .\right.
\end{aligned}
$$

In the limit (b) we find $n(\rho)$ by solving Eqs. (1) and (2) numerically. The numerical solution shown in Fig. 2(d) interpolates between the regimes of weak and strong graphene doping, as follows:

$$
\begin{aligned}
& n^{I} \approx\left\{\begin{array}{c}
\sqrt{\frac{\tilde{A} \chi \rho}{2 \pi e^{2}}}, \quad \rho \ll \rho_{*}^{I}, \\
\tilde{n}_{*}^{I}, \quad \rho \gg \rho_{*}^{I} ;
\end{array}\right. \\
& \tilde{n}_{*}^{I}=\frac{\tilde{A} \tilde{\gamma}_{A}}{\left(1+\sqrt{1+\frac{\tilde{\gamma}_{A}}{\gamma_{d}}}\right)^{2}}, \quad \tilde{\gamma}_{A}=\frac{4 \tilde{A}}{\pi \hbar^{2} v^{2}}, \\
& \rho_{*}^{I}=\frac{2 \pi e^{2} \tilde{A} \tilde{\gamma}_{A}^{2}}{\chi\left(1+\sqrt{1+\frac{\tilde{\gamma}_{A}}{\gamma_{d}}}\right)^{4}} .
\end{aligned}
$$

The responsivity, $\left.r\right|_{n_{g}=0}$ of MLG in SiC/G in the limit (b) can be described using Eq. (6), but with $\gamma_{A}$ replaced by $\widetilde{\gamma}_{A}$ and the upper/lower limits corresponding to $\rho \ll \rho_{*}^{I}$ and $\rho \gg \rho_{*}^{I}$, respectively.

BLG has the spectrum ${ }^{8}$ with conduction $(+)$ and valence $(-)$ bands, which, in the vicinity of the Brillouin zone corners, are described by
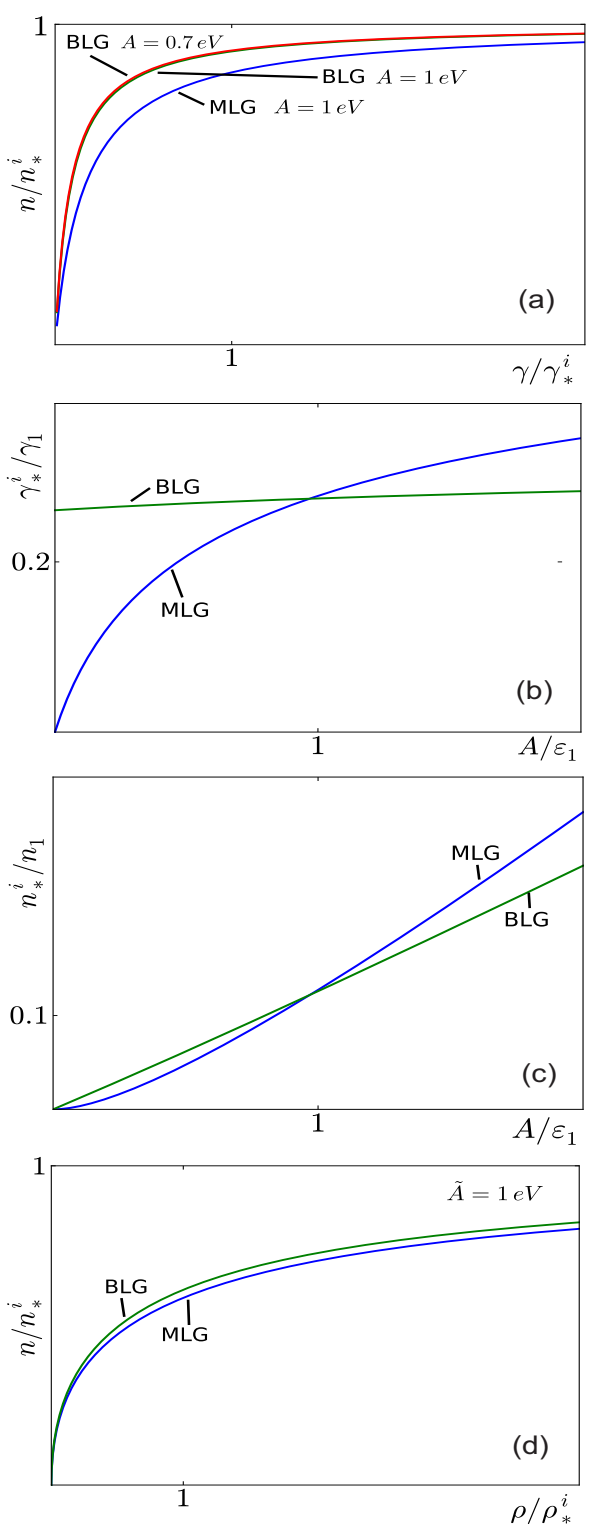

FIG. 2. (Color online) Comparison between charge transfer from $\mathrm{SiC}$ to MLG and BLG, with $A$ measured in units of split-band energy in BLG, $\varepsilon_{1}$ $=0.4 \mathrm{eV}, d=0.3 \mathrm{~nm}$ for MLG and $d=0.5 \mathrm{~nm}$ for BLG. (a) Electron concentration in graphene $n$ dominated by charge transfer from surface states. (b) Values $\gamma_{*}$ of the surface DoS at which $n(\gamma)$ saturates. (c) Saturation density value as a function of $n_{*}$, in units of $n_{1}$. (d) Electron bulk donors density $\rho$ for $\gamma=0$.

$$
\varepsilon_{s \pm}(p)= \pm\left(\sqrt{\frac{\varepsilon_{1}^{2}}{4}+v^{2} p^{2}}+\frac{s \varepsilon_{1}}{2}\right)
$$

where $\varepsilon_{1}$ is the interlayer coupling and $s$ distinguishes between pairs of degenerate $(s=-1)$ and split $(s=1)$ bands. The Fermi level in n-doped BLG with sheet electron density $n$ is determined as

$$
\varepsilon_{F}=\left\{\begin{array}{ll}
\sqrt{\frac{\varepsilon_{1}^{2}}{4}+\pi \hbar^{2} v^{2} n}-\frac{\varepsilon_{1}}{2}, & n<n_{1} \\
\hbar v \sqrt{\frac{\pi n}{2}}, & n>n_{1}
\end{array},\right.
$$

where $n_{1}=2 \varepsilon_{1}^{2} /\left(\pi \hbar^{2} v^{2}\right) \approx 3 \times 10^{13} \mathrm{~cm}^{-2}$.

In the limit (a), this gives 


$$
n^{I I}=\frac{2 A\left(A+\varepsilon_{1}\right) /\left(\pi \hbar^{2} v^{2}\right)}{1+\frac{\tilde{\gamma}_{1}}{\gamma_{d}}+\frac{\tilde{\gamma}_{1}}{\gamma}+\sqrt{\left(1+\frac{\gamma_{1}}{\gamma_{d}}+\frac{\gamma_{1}}{\gamma}\right)^{2}+\frac{\gamma_{A}}{\gamma_{d}}+\frac{\gamma_{A}}{\gamma}}},
$$

for $n^{I I}<n_{1}$ and $n_{g}=0$. Here, $\gamma_{1}=\varepsilon_{1} / \pi \hbar^{2} v^{2}$ and $\tilde{\gamma}_{1}=\gamma_{1}$ $+\gamma_{A} / 2$. For larger densities, $n^{I I}>n_{1}$,

$$
n^{I I}=\frac{8 A^{2}}{\pi \hbar^{2} v^{2}} \frac{1}{\left(1+\sqrt{1+\frac{2 \gamma_{A}}{\gamma}+\frac{2 \gamma_{A}}{\gamma_{d}}}\right)^{2}},
$$

which resembles Eq. (3) for MLG, but with $\gamma_{A} \rightarrow 2 \gamma_{A}$.

Similarly to MLG, the density in BLG on SiC saturates upon the increase in surface DoS of donors. The crossover to the saturated density, $n_{*}^{I I}(A)=A \gamma_{*}^{I I}(A)$, occurs at

$$
\gamma_{*}^{I I}= \begin{cases}\frac{2 \gamma_{A}}{\left(1+\sqrt{1+\frac{2 \gamma_{A}}{\gamma_{d}}}\right)^{2}}, & \frac{2 \gamma_{1}}{\gamma_{d}}+1<\frac{\gamma_{A}}{4 \gamma_{1}} \\ \frac{\frac{\gamma_{A}}{2}+2 \gamma_{1}}{1+\frac{\gamma_{A}}{2 \gamma_{d}}+\frac{\gamma_{1}}{\gamma_{d}}+\sqrt{\left(1+\frac{\gamma_{1}}{\gamma_{d}}\right)^{2}+\frac{\gamma_{A}}{\gamma_{d}}},} & \frac{2 \gamma_{1}}{\gamma_{d}}+1>\frac{\gamma_{A}}{4 \gamma_{1}}\end{cases}
$$

The dependence of $n^{I I}(\gamma), \gamma_{*}^{I I}(A)$, and $n_{*}^{I I}(A)$ on the relative size of the band splitting $\varepsilon_{1}$, and the graphene-surface donors work function $A$ is shown in Figs. 2(a)-2(c). Responsivity of the BLG to the gate voltage is high or low, depending on whether the saturation regime for the carrier density is reached, or not. For $\gamma \ll \gamma_{*}^{I I}, r \approx 1$. For $\gamma \gg \gamma_{*}^{I I}$,

$$
r \approx\left\{\begin{array}{cc}
1-\frac{1}{\sqrt{1+\frac{2 \gamma_{A}}{\gamma_{d}}},} \quad & \frac{2 \gamma_{1}}{\gamma_{d}}+1<\frac{\gamma_{A}}{4 \gamma_{1}} \\
1-\frac{1}{\sqrt{\left(1+\frac{\gamma_{1}}{\gamma_{d}}\right)^{2}+\frac{\gamma_{A}}{\gamma_{d}}}}, & \frac{2 \gamma_{1}}{\gamma_{d}}+1>\frac{\gamma_{A}}{4 \gamma_{1}}
\end{array} .\right.
$$

In the limit (b), when $n$ is determined by charge transfer from bulk donors in $\mathrm{SiC}$,

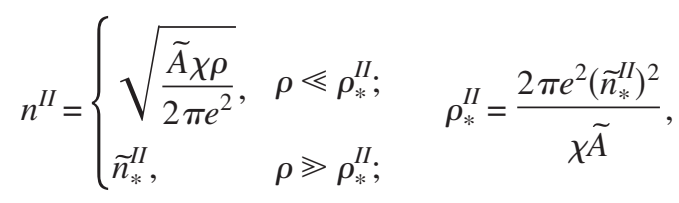

with $\widetilde{n}_{*}^{I I}=n_{*}^{I I}(\widetilde{A})$, and responsivity $r \approx 1$ of BLG requires that $\rho \ll \rho_{*}^{I I}$, whereas for $\rho \gg \rho_{*}^{I I}$ responsivity is described by the same limits as in Eq. (8), with $\gamma_{A}$ replaced by $\tilde{\gamma}_{A}$.

Independently of the number of layers, the gate voltage $V_{g}^{*}$ needed to reach the neutrality point in graphene controlled by the top gate with mutual capacitance $C$ is

$$
V_{g}^{*} \approx \frac{e}{C} \max \left(\frac{A}{\gamma^{-1}+\gamma_{d}^{-1}}, \frac{2 \tilde{A} \gamma_{d}}{1+\sqrt{1+\frac{8 \pi e^{2} \tilde{A} \gamma_{d}^{2}}{\chi \rho}}}\right)
$$

In summary, we calculated charge transfer from $\mathrm{SiC}$ to epitaxial graphene. In the case when the charge transfer is dominated by donors on the surface of $\mathrm{SiC}$ with $A \sim 1 \mathrm{eV}$ or donors in the bulk of $\mathrm{SiC}$ with $\widetilde{A} \sim 1 \mathrm{eV},{ }^{11}$ we estimate that the saturation density of n-type doping of MLG is 1 $\times 10^{13} \mathrm{~cm}^{-2}$, which corresponds to $\varepsilon_{F} \approx 0.4 \mathrm{eV}$ (for $d$ $\approx 0.3 \mathrm{~nm}$ ). This value of carrier density occurs when the donor volume density is $\rho>\rho_{*}^{I} \sim 1 \times 10^{19} \mathrm{~cm}^{-3}$ [we use $\chi$ $\sim 10$ for $6 \mathrm{H} \mathrm{SiC} \mathrm{(Ref.} \mathrm{12)]} \mathrm{or} \mathrm{the} \mathrm{surface} \mathrm{states} \mathrm{have} \mathrm{DoS}$ $\gamma>\gamma_{*}^{I} \sim 1 \times 10^{13} \mathrm{~cm}^{-2} \mathrm{eV}^{-1}$. For lesser doping of $\mathrm{SiC}, \gamma$ $<\gamma_{*}$ and $\rho<\rho_{*}$, one should use the larger of the estimates from Eqs. (4) and (7) This can be compared to the data reported in the recent studies of epitaxial graphene indicating a substantial initial level of n-type doping of $\mathrm{SiC} / \mathrm{G}$, very often ${ }^{4}$ as high as $1 \times 10^{13} \mathrm{~cm}^{-2}$. However, some particular growth processes produce $\mathrm{SiC} / \mathrm{G}$ with a much lower doping level, ${ }^{5,13}$ indicating that efficient annealing of donors on and near the $\mathrm{SiC}$ surface is possible.

We thank T. Seyller and R. Yakimova for discussions. This work was supported by EPSRC under Grant Nos. EP/ G041954 and EU-FP7 ICT STREP Concept Graphene.

${ }^{1}$ A. K. Geim, Science 324, 1530 (2009).

${ }^{2}$ T. Ohta, A. Bostwick, T. Seyller, K. Horn, and E. Rotenberg, Science 313, 951 (2006); A. Bostwick, T. Ohta, T. Seyller, K. Horn, and E. Rotenberg, Nat. Phys. 3, 36 (2007); T. Ohta, A. Bostwick, J. L. McChesney, T. Seyller, K. Horn, and E. Rotenberg, Phys. Rev. Lett. 98, 206802 (2007). ${ }^{3}$ C. Virojanadara, M. Syvajarvi, R. Yakimova, L. I. Johansson, A. A. Zakharov, and T. Balasubramanian, Phys. Rev. B 78, 245403 (2008); G. Gu, S. Nie, R. M. Feenstra, R. P. Devaty, W. J. Choyke, W. K. Chan, and M. G. Kane, Appl. Phys. Lett. 90, 253507 (2007); Y. Q. Wu, P. D. Ye, M. A. Capano, Y. Xuan, Y. Sui, M. Qi, J. A. Cooper, T. Shen, D. Pandey, G. Prakash, and R. Reifenberger, ibid. 92, 092102 (2008); J. Kedzierski, P.-L. Hsu, P. Healey, P. Wyatt, C. Keast, M. Sprinkle, C. Berger, and W. A. de Heer, IEEE Trans. Electron Devices 55, 2078 (2008).

${ }^{4}$ K. V. Emtsev, A. Bostwick, K. Horn, J. Jobst, G. L. Kellogg, L. Ley, J. L. McChesney, T. Ohta, S. A. Reshanov, J. Rohrl, E. Rotenberg, A. K. Schmid, D. Waldmann, H. B. Weber, and T. Seyller, Nature Mater. 8, 203 (2009); C. Coletti, C. Riedl, D. S. Lee, B. Krauss, L. Patthey, K. von Klitzing, J. H. Smet, and U. Starke, Phys. Rev. B 81, 235401 (2010); J. Jobst, D. Waldmann, F. Speck, R. Hirner, D. K. Maude, T. Seyller, and H. B. Weber, ibid. 81, 195434 (2010); J. S. Moon, D. Curtis, S. Bui, M. Hu, D. K. Gaskill, J. L. Tedesco, P. Asbeck, G. G. Jernigan, B. L. VanMil, R. L. Myers-Ward, C. R. Eddy, P. M. Campbell, and X. Weng, IEEE Electron Device Lett. 31, 260 (2010).

${ }^{5}$ A. Tzalenchuk, S. Lara-Avila, A. Kalaboukhov, S. Paolillo, M. Syvajarvi, R. Yakimova, O. Kazakova, T. J. B. M. Janssen, V. Fal'ko, and S. Kubatkin, Nat. Nanotechnol. 5, 186 (2010).

${ }^{6}$ P. N. First, W. A. de Heer, T. Seyller, C. Berger, J. A. Stroscio, and J. S. Moon, MRS Bull. 35, 296 (2010).

${ }^{7}$ Y. M. Lin, K. A. Jenkins, A. Valdes-Garcia, J. P. Small, D. B. Farmer, and P. Avouris, Nano Lett. 9, 422 (2009).

${ }^{8}$ E. McCann and V. I. Fal'ko, Phys. Rev. Lett. 96, 086805 (2006).

${ }^{9}$ E. V. Castro, K. S. Novoselov, S. V. Morozov, N. M. R. Peres, J. M. B. Lopes dos Santos, J. Nilsson, F. Guinea, A. K. Geim, and A. H. Castro Neto, Phys. Rev. Lett. 99, 216802 (2007); J. B. Oostinga, H. B. Heersche, L. Liu, A. F. Morpurgo, and L. M. K. Vandersypen, Nature Mater. 7, 151 (2007); Y. Zhang, T. T. Tang, C. Girit, Z. Hao, M. C. Martin, A. Zettl, M. F. Crommie, Y. Ron Shen, and F. Wang, Nature (London) 459, 820 (2009); B. N. Szafranek, D. Schall, M. Otto, D. Neumaier, and H. Kurz, Appl. Phys. Lett. 96, 112103 (2010).

${ }^{10}$ J. Bardeen, Phys. Rev. 71, 717 (1947).

${ }^{11}$ S. Sonde, F. Giannazzo, V. Raineri, R. Yakimova, J. R. Huntzinger, A. Tiberj, and J. Camassel, Phys. Rev. B 80, 241406 (2009).

${ }^{12}$ L. Patrick and W. J. Choyke, Phys. Rev. B 2, 2255 (1970).

${ }^{13}$ S. Weingart, C. Bock, U. Kunze, F. Speck, Th. Seyller, and L. Ley, Appl. Phys. Lett. 95, 262101 (2009). 